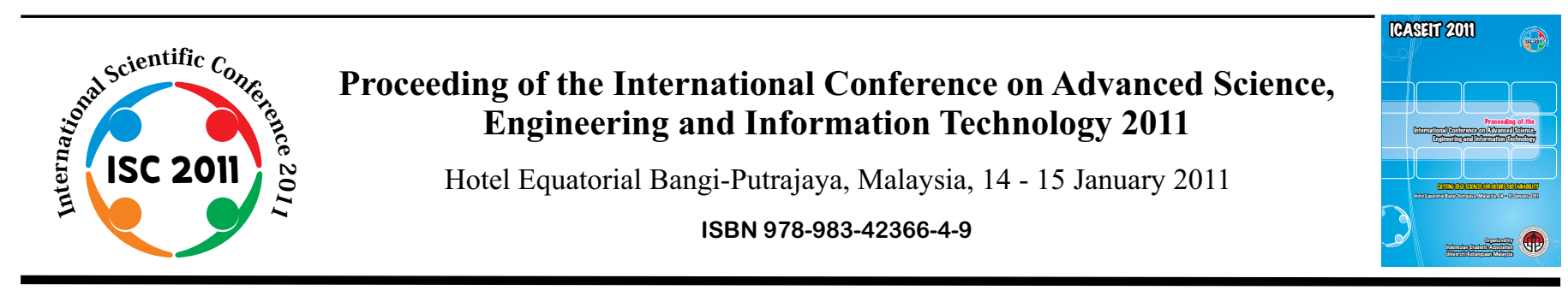

\title{
Shape Transformation of Left Ventricle Wall for Cardiac Global Hypokinetic Evaluation
}

\author{
Adhi Harmoko Saputro ${ }^{\# 1}$, Mohd. Marzuki Mustafa ${ }^{\# 2}$, Aini Hussain ${ }^{\# 3}$, \\ Oteh Maskon ${ }^{* 4}$ and Ika Faizura Mohd Nor ${ }^{* 5}$ \\ * Department of Electrical, Electronic and Systems Engineering \\ Universiti Kebangsaan Malaysia, Bangi 43600, Malaysia \\ Email: 'adhi.harmoko@ui.ac.id, '2marzuki@vlsi.eng.ukm.my,3aini@vlsi.eng.ukm.my \\ *The Cardiac Care Unit, Medical Center \\ Universiti Kebangsaan Malaysia, Kuala Lumpur 56000, Malaysia \\ Email: ${ }^{4}$ auajwad@yahoo.com, ${ }^{5}$ azuzayz@yahoo.ie
}

\begin{abstract}
A method to evaluate left ventricular ( $\mathrm{LV})$ wall motion in two-dimensional (2D) echocardiographic image is proposed. It is used to investigate posterior and anterior septum wall shape change while pumping the blood in human cardiac. Shape transformation parameters of myocardial wall boundary were extracted to indicate the LV wall movement from end diastole to end systole. Quantitative parameters represent the movement of $L V$ wall segment whatever translation, rotation, expansion or combination of them. Initial myocardial boundary is drawn manually on end diastole cycle and then tracked to all frames by computing the speckle motion estimation in each frame of cardiac cycle. The motion vector of myocardial boundary movement is computed using warping optical flow on wavelet multi-resolution. The method was applied to parasternal long axis view of $2 \mathrm{D}$ echocardiographic from normal subject and patients with global hypokinetic. The results show that quantitative evaluation parameters gave a potential indication in evaluating and diagnosing myocardial wall motion abnormalities.
\end{abstract}

Keywords—myocardial motion, echocardiographic, shape transformation, motion estimation.

\section{INTRODUCTION}

Currently, the left ventricular (LV) wall motion can be analyzed on three schemes namely qualitative, semiquantitative and quantitative assessment. Eyeball assessment, normal-abnormal comparison and normal-hypokineticakinetic-dyskinetic scheme are classified as quantitative assessment. Wall motion score index is classified as semiquantitative assessment. Whereas for accurate assessment can be done by measuring left ventricle regional quantitatively which analyze global function as a percentage of normal condition such as radial and fractional shortening [1], fractional cavity area change, chordal centerline analysis, wall velocity, myocardial displacement [2], myocardial gradient, strain and strain rate $[3,4]$.

The term hypokinetic is used for the motor function of muscle that performs below normal condition. In cardiac disease, hypokinetic is classified as ischemic disease which caused by a chronic lack of oxygen in coronary artery. Hypokinetic is one of dysfunction of the left ventricle. It means some segments of the myocardium are in low contraction function. Whereas, the term global hypokinetic is used to indicate all segment of the myocardium in low contraction function

In the previous works, the motion estimation on echocardiographic image using optical flow and wavelet decomposition has been described [5]. In this technique, two dimensional motion vectors was generated accurately on parasternal long axis view of human cardiac for both case normal subject and hypokinetic patients. The abnormalities evaluation of left ventricle wall motion is hardly difficult tasks, due to it is performed visually based on the motion vector near the LV wall. To make it useful for diagnosis purposes, it is important to find some quantitative representation for the wall vector motion.

In this work, we proposed shape transformation parameters for the quantitative evaluation of the LV wall motion. The shape transformation parameters are obtained by finding transformation matrix between initial boundary wall and final boundary wall. In particular, we investigate the ability of 
shape transformation parameters to assess the shape pattern in global hypokinetic case. This assessment is performed on $2 \mathrm{D}$ echocardiographic image from twelve normal subject and three patients with global hypokinetic.

\section{Methodology}

In this section, the detail of methodology of cardiac global kinetic evaluation using LV wall shape transformation is explained.

\section{A. Cardiac shape transformation}

Let $X_{o}(x, y)$ be the initial position of any point in first frame of a image sequence where $x$ and $y$ indicate the horizontal and vertical position in Cartesian coordinate. This point can be transformed into a new point using transformation functional form $T$ as follow

$$
X_{t}=T \cdot X_{o}
$$

where $X_{o}$ is initial point of $x_{o}, y_{o}$ and $X_{t}$ is final point of $x_{t}, y_{t}$.

In shape transformation, the initial position $X_{o}$ can be consist a number of points which create a shape and can be written as follow

$$
\left[X_{1} X_{2} X_{3} \cdots X_{n}\right]_{t}=T\left[X_{1} X_{2} X_{3} \cdots X_{n}\right]_{o}
$$

In the $2 \mathrm{D}$ geometric transformation, the functional form $T$ can be represented using a $2 \times 2$ matrix for most common transformation such as linier, rotation, scaling, shearing, reflection and orthogonal (see Table 1). The complex transformation can be generated by combine several transformations using simple matrix multiplication of each functional form.

TABLE I

$2 \times 2$ MATRIX FOR MOST COMMON TRANSFORMATION

\begin{tabular}{|l|c|l|}
\hline \multicolumn{1}{|c|}{ Transformation } & $\begin{array}{c}\text { Functional form } \\
T\end{array}$ & \multicolumn{1}{|c|}{ Information } \\
\hline Linier & {$\left[\begin{array}{ll}x & 0 \\
0 & y\end{array}\right]$} & $\begin{array}{l}\mathrm{x}=\text { horizontal } \\
\mathrm{y}=\text { vertical }\end{array}$ \\
\hline $\begin{array}{l}\text { Counter-clockwise } \\
\text { rotation }\end{array}$ & {$\left[\begin{array}{cc}\cos \theta & \sin \theta \\
-\sin \theta & \cos \theta\end{array}\right]$} & $\theta=$ rotation angle \\
\hline Clockwise rotation & {$\left[\begin{array}{cc}\cos \theta & -\sin \theta \\
\sin \theta & \cos \theta\end{array}\right]$} & $\theta=$ rotation angle \\
\hline Scaling & {$\left[\begin{array}{cc}S_{x} & 0 \\
0 & S_{y}\end{array}\right]$} & $\mathrm{S}_{\mathrm{x}}=$ horizontal \\
& {$\left[\begin{array}{ll}1 & k \\
0 & 1\end{array}\right]$} & Parallel to the $x$ axis \\
\hline Shearing & {$\left[\begin{array}{ll}1 & 0 \\
k & 1\end{array}\right]$} & Parallel to the $y$ axis \\
\hline
\end{tabular}

In general, the human cardiac has several motion directions that occurs in the cardiac cycle such as radial movement (expansion or contraction), horizontal rotation (clockwise or counter clockwise) and vertical rotation (upward or downward) [6]. Combining this motion directions into a standardized division of the left ventricle according to the American Heart Association [7], motions that depicted using the short axis view have radial and horizontal motion. Whereas, radial motions is depicted using the long axis view. Figure 1 shows the visualisation of motion direction in each cardiac view.

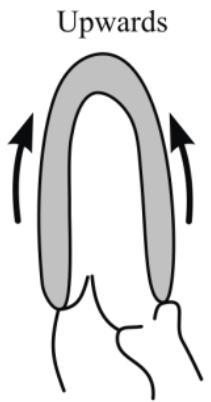

Expansion

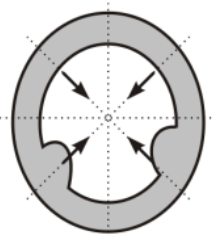

Clockwise

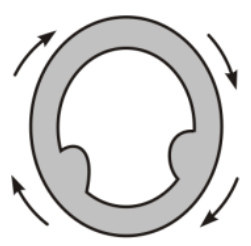

Downwards
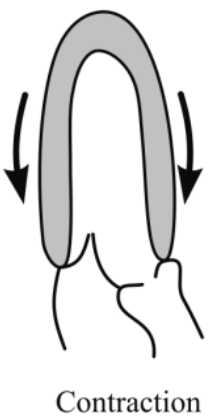

b

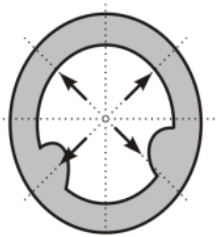

Conter-clockwise

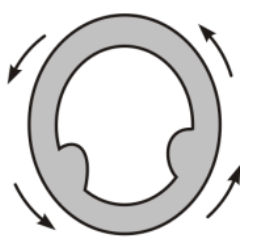

c.

Fig. 1. The visualisation of motion direction in (a) long axis view (upwards or downwards), (b) and (c) short axis view (expansion or contraction and clockwise or counter-clockwise)

The shape change as shown in figure 1 that caused by cardiac muscle motion can be represented as a $2 \mathrm{D}$ geometric transformation. Expansion and contraction motion can be represented as linier or scaling transformation whereas upwards and downwards motion can be generated by combination of rotation and scaling transformation. This representation of shape transformation and shape change of myocardium inspired a method that use shape transformation parameter to evaluate the wall motion abnormalities.

\section{B. Computing transformation parameters}

Transformation parameters of myocardial boundary that used to evaluate the wall motion abnormalities are generated by computing the shape transformation of myocardial wall in end systole and in end diastole. Moreover these parameters are used to describe the left ventricle contraction performance while pumping the blood. Scaling value in transformation matrix can be substituted the left ventricular ejection fraction (LVEF) that has established for cardiac abnormalities evaluation as in [8].

Detail of transformation parameters computation is described by Figure 2. Computation process of these parameters is performed as follow. A set motion vector of all frames was generated using optical flow on wavelet multiresolution [5]. This motion estimation was used due to its accuration in estimating $u$ and $v$ velocity in speckle noisy image. Cardiologist should draw the initial boundary by a number of points along the septum and the posterior in first frame of sequence (end diastole). The final boundary was 
formed by tracking initial point to last frame of sequence (end systole) by computing the point movement based on the velocity that generated by the motion estimation. Finally, there are two shapes of myocardial boundary, diastole wall shape (first frame) and systole wall shape (last frame).

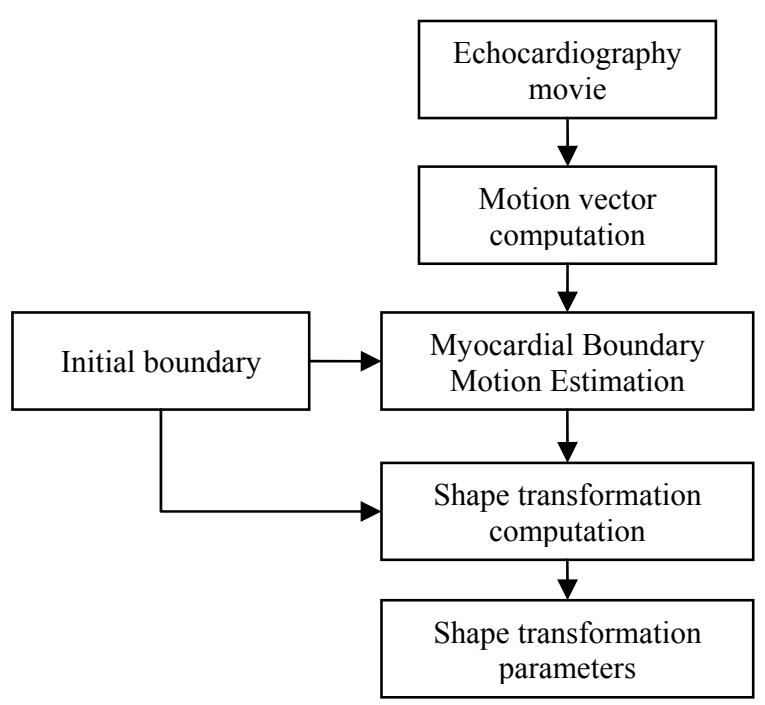

Fig. 2 A flowchart of shape transformation parameters computation in left ventricle wall on $2 \mathrm{D}$ echocardiographic image

Transformation parameters of both shapes is computed by arrange the points as follow

$$
\left[X_{1} X_{2} X_{3} \cdots X_{n}\right]_{t}-T\left[X_{1} X_{2} X_{3} \cdots X_{n}\right]_{o}=0
$$

where $\left[X_{1} X_{2} X_{3} \cdots X_{n}\right]_{t}$ is a set point in end systole and $\left[X_{1} X_{2} X_{3} \cdots X_{n}\right]_{o}$ is a set point in end diastole. Both of set points must be same in dimension.

Equation 3 can be solved using least square equation solution to find the transformation matrix $\mathrm{T}$ as follow

$$
T \Rightarrow\left[\begin{array}{ll}
S_{x} & S_{o} \\
S_{o} & S_{y}
\end{array}\right]
$$

where $S_{x}$ is horizontal scaling factor, $S_{y}$ is vertical scaling factor and $S_{o}$ should be near zero $\left(S_{o} \approx 0\right)$.

\section{RESULT AND DISCUSSION}

The experiment result of the cardiac evaluation with normal subject, patient with global hypokinetic, shape transformation computing and physical meaning of parameters is presented in this section.

The echocardiographic data were acquired from twelve normal subjects and three patients with global hypokinetic using Acuson Squoia C512 Ultrasound Machine at Cardiac Care Unit UKM Medical Centre. These echocardiographic data had a width and height of $384 \times 287$ pixels and recorded in dicom medical image format to preserve the speckle information.

\section{A. Myocardial Boundary Tracking}

Myocardial boundary tracking from end diastole to end systole was performed based on the velocity motion field that computed using generated using optical flow on wavelet multi-resolution [5]. Figure 3(a) shows the motion vector of the normal subject that superimposed to its first frame of echocardiographic image sequences. Moreover figure 3(b) shows the example of initial boundary (yellow line) and the final boundary (green line) of global hypokinetic patient that superimposed to its last frame of echocardiographic image.

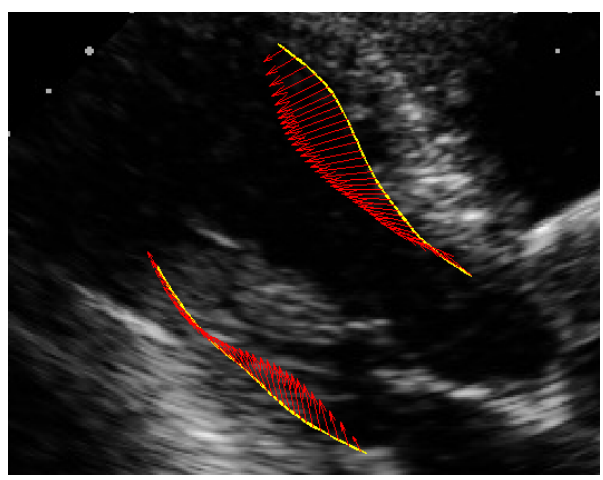

(a)

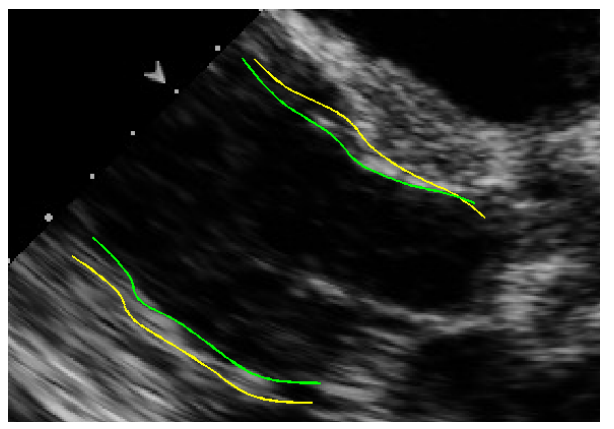

(b)

Fig. 3. (a) Motion vector visualisation along left ventricle wall on norma subject. (b) Initial boundary in end diastole (yellow line) was tracked to final boundary in end systole (green line)

The accuracy of motion tracking algorithm and motion estimation has been evaluated on the real clinical dataset by expert cardiologist qualitatively and on simulated echocardiographic image quantitatively [9]. The result expresses that the motion technique has a good performance in tracking an object on the speckled image.

\section{B. Shape Transformation Parameter Result}

The shape transformation parameters $\left(S_{x}\right.$ and $\left.S_{y}\right)$ have been computed form twelve normal subjects and three patients with global hypokinetic. The average and standard deviation of these data are plotted as shown in figure 4 . The parameter $\mathrm{S}$ is a scaling factor of both direction $\mathrm{x}$ and $\mathrm{y}$. The data is grouped by the normal subject and global hypokinetic patients.

The result shown in figure 4 shows the comparison of normal segment and global hypokinetic segment. Considering the shape transformation parameters, the average and standard deviation yielded a significant difference between two groups for both parameters. The horizontal scaling factor showed the significant differences between normal $\left(S_{x}=0.88 \pm 0.02\right)$ 
versus global hypokinetic $\left(S_{x}=0.96 \pm 0.01\right)$. The vertical scaling factor also resulted in significant differences between normal $\left(S_{y}=0.84 \pm 0.05\right)$ versus global hypokinetic $\left(S_{x}=0.95\right.$ $\pm 0.04)$.

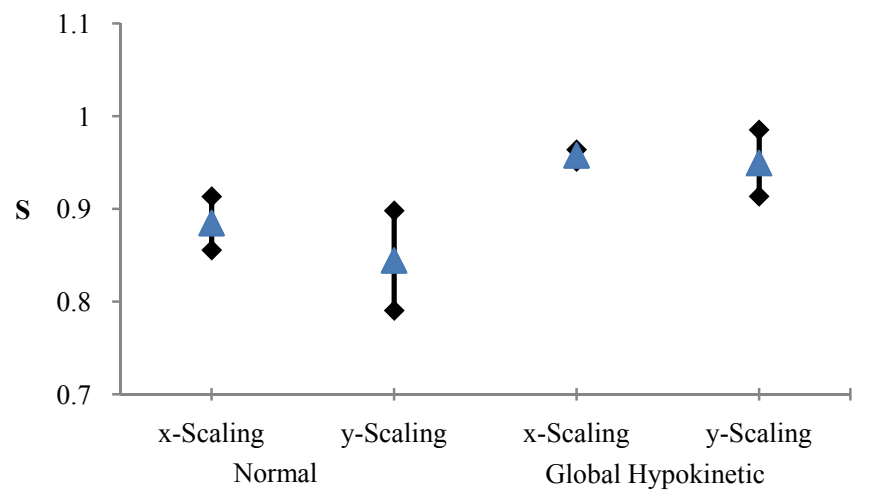

Fig. 4. Mean and standard deviation for shape transformation (T) in $\mathrm{x}$ and $\mathrm{y}$ directions in normal and global hypokinetic cases.

The shape transformation parameters $\left(S_{x}\right.$ and $\left.S_{y}\right)$ have physical meaning regarding the movement of myocardial segment. These parameters are scaling factor of shape transformation. If the scaling factor is lower than one, the shape is smaller than the previous one. Otherwise the scaling factor is higher than one; the shape is bigger than the previous one. While the blood pumping process at the end of diastole to the end of systole, the volume of left ventricle is decline. In other word, the myocardial boundary is shrinking in this process. The normal subject should have bigger shrinking than the hypokinetic patient. Figure 4 show that the normal subjects have $\mathrm{S}$ parameters more than global hypokinetic. This agrees with the left ventricular ejection fraction (LVEF) which the normal subject have the LVEF at $69.6+7.4 \%$ for men and $67.8+7.2 \%$ for woman [10] whereas the abnormal contraction function has the LVEF less than $40 \%$.

\section{CONCLUSIONS}

In this paper, a method to evaluate cardiac global hypokinetic using shape transformation parameters has been presented. This method exploits the transformation matrix of myocardial boundary shape change as a reference to detect left ventricular abnormalities especially in global hypokinetic patient. The experiment result obtained with real clinical data from normal subject and patient with global hypokinetic express the ability of the method to evaluate the left ventricle contraction abnormalities. It may also be interesting that quantitative evaluation parameters gave a potential indication in evaluating and diagnosing all myocardial wall motion abnormalities.

\section{ACKNOWLEDGMENT}

The authors would like to acknowledge Universiti Kebangsaan Malaysia (Project code UKM-GUP-TKP- 08-24080) for the financial support awarded for this research.

\section{REFERENCES}

[1] E. W. Remme, A. A. Young, K. F. Augenstein, B. Cowan, and P. J. Hunter, "Extraction and quantification of left ventricular deformation modes," IEEE Transactions on Biomedical Engineering, vol. 51, pp. 1923-1931, 2004.

[2] L. Dougherty, J. C. Asmuth, A. S. Blom, L. Axel, and R. Kumar, "Validation of an optical flow method for tag displacement estimation," IEEE Transactions on Biomedical Engineering, vol. 18, pp. 359-363, 1999.

[3] C. Hao, T. Varghese, P. S. Rahko, and J. A. Zagzebski, "Evaluation of Cardiac Dyssynchrony Using Strain Imaging," in Ultrasonics Symposium, 2007. IEEE, pp. 1941-1944, 2007.

[4] M. Sühling, M. Arigovindan, C. Jansen, P. Hunziker, and M. Unser, "Myocardial Motion and Strain Rate Analysis from Ultrasound Sequences " Complex Motion - Lecture Notes in Computer Science, vol. 3417, pp. 177-189, 2007.

[5] A. H. Saputro, M. M. Mustafa, A. Hussain, O. Maskon, and I. F. M. Nor, "Myocardial motion analysis using optical flow and wavelet decomposition," in 2010 6th International Colloquium on Signal Processing and Its Applications (CSPA), pp. 1-5, 2010.

[6] M. d. S. a. Rebelo, A. K. H. Aarre, K.-L. Clemmesen, S. C. S. Brand ao, M. C. Giorgi, J. e. Cl'audioMeneghetti, and a. A. Gutierrez, "Determination of Three-Dimensional Left VentricleMotion to Analyze Ventricular Dyssyncrony in SPECT Images," EURASIP Journal on Advances in Signal Processing, vol. 2010, 2010.

[7] Manuel D. Cerqueira, Neil J. Weissman, Vasken Dilsizian, Alice K. Jacobs, Sanjiv Kaul, Warren K. Laskey, Dudley J. Pennell, John A. Rumberger, Thomas Ryan, and M. S. Verani, "Standardized Myocardial Segmentation and Nomenclature for Tomographic Imaging of the Heart " The American Heart Association, vol. 105, pp. 539-542, 2002 .

[8] E. A. Bocchi, A. Esteves-Filho, G. Bellotti, F. Bacal, L. F. Moreira, N Stolf, and J. F. Ramires, "Left ventricular regional wall motion, ejection fraction, and geometry after partial left ventriculectomy. Influence of associated mitral valve repair," Eur J Cardiothorac Surg, vol. 18, pp. 458-465, October 1, 20002000.

[9] A. H. Saputro, M. M. Mustafa, A. Hussain, O. Maskon, and I. F. M. Nor, "Development of A Quantitative Evaluation Motion Estimation Technique using Simulated Echocardiograohy Image Sequence," in Regional Engineering Postgraduate Conference (EPC) 2010, Bangi, Malaysia, pp. 417-423, 2010.

[10] T. Kuznetsova, L. Herbots, T. Richart, J. D'hooge, L. Thijs, R. H. Fagard, M.-C. Herregods, and J. A. Staessen, "Left ventricular strain and strain rate in a general population," European Heart Journal, vol. 29, pp. 2014-2023, August 20082008. 